University of Nebraska - Lincoln

DigitalCommons@University of Nebraska - Lincoln

Faculty Publications from the Department of Engineering Mechanics

Mechanical \& Materials Engineering,

Department of

2004

\title{
Continuum Modeling of Cell Membranes
}

Eveline Baesu

University of Nebraska - Lincoln, ebaesu2@unl.edu

R. E. Rudd

Lawrence Livermore National Laboratory, 7000 East Ave, Livermore, CA

J. Belak

Lawrence Livermore National Laboratory, 7000 East Ave, Livermore, CA

M. McElfresh

Materials Research Institute, 7000 East Ave, Livermore, CA

Follow this and additional works at: https://digitalcommons.unl.edu/engineeringmechanicsfacpub

Part of the Mechanical Engineering Commons

Baesu, Eveline; Rudd, R. E.; Belak, J.; and McElfresh, M., "Continuum Modeling of Cell Membranes" (2004). Faculty Publications from the Department of Engineering Mechanics. 52.

https://digitalcommons.unl.edu/engineeringmechanicsfacpub/52

This Article is brought to you for free and open access by the Mechanical \& Materials Engineering, Department of at DigitalCommons@University of Nebraska - Lincoln. It has been accepted for inclusion in Faculty Publications from the Department of Engineering Mechanics by an authorized administrator of DigitalCommons@University of Nebraska - Lincoln. 


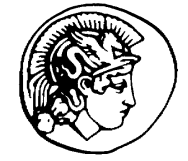

PERGAMON
SCIEnce@Direct.

International Journal of Non-Linear Mechanics 39 (2004) 369-377
NON-LINEAR MECHANICS

\title{
Continuum modeling of cell membranes
}

\author{
E. Baesu ${ }^{\mathrm{a}, *}$, R.E. Rudd ${ }^{\mathrm{b}}$, J. Belak ${ }^{\mathrm{b}}$, M. McElfresh ${ }^{\mathrm{c}}$ \\ ${ }^{a}$ Department of Engineering Mechanics, University of Nebraska-Lincoln, W304 Nebraska Hall, Lincoln, NE 68588-0526, USA \\ ${ }^{\mathrm{b}}$ Lawrence Livermore National Laboratory, 7000 East Ave, Livermore, CA 94550, USA \\ ${ }^{\mathrm{c}}$ Materials Research Institute, 7000 East Ave, Livermore, CA 94550, USA
}

Received 9 April 2002; accepted 21 October 2002

\begin{abstract}
In this paper, we develop a finite-deformation model for cell membranes with a view toward characterizing the local mechanical response of membranes in atomic force microscope (AFM) experiments. The membrane is modeled as a 2-D fluid continuum endowed with bending resistance. The general theory is used to obtain equations that describe axisymmetric equilibrium states. The membrane is assumed to enclose a fluid medium, which transmits hydrostatic pressure to the membrane, and a point load is applied at the pole to simulate an AFM probe. Both types of loading are associated with a potential and the problem is then cast in a variational setting. The equilibrium equations and boundary conditions are obtained by applying standard variational procedures, resulting in a pair of coupled fourth-order differential equations to be solved for the shape of the meridian. Further refinements associated with global constraints on the enclosed volume and contact with a rigid substrate are introduced, and a solution strategy is proposed which relies on an iterative scheme for calculating the associated Lagrange multipliers.
\end{abstract}

\section{Introduction}

The development of atomic force microscopy (AFM) and its generalizations, collectively known as scanning probe microscopy, has provided dramatically new insights into many solid materials systems including biological systems (see [1] for example and the references cited therein). These techniques offer a non-destructive characterization of the mechanical and chemical properties of systems on the nanoscale. The experimental methodologies have proven especially effective in the characterization of hard

\footnotetext{
* Corresponding author. Tel.: +1-402-472-2382; fax: +1-402472-8292.

E-mail address: ebaesu@unlserve.unl.edu (E. Baesu).
}

solid-state systems, such as the surface morphology of semiconductors. Soft materials, such as biological membranes, have proven more difficult. Recent advances have enabled AFM to be used to image living cells with a resolution of a few nanometers, but mechanical characterization of soft biological systems by AFM typically suffers from irreproducibility [3].

Optical tweezer techniques have also proven to be extremely useful in the study of biological systems on the nanoscale (see for example [2] and the references cited therein). They have been used extensively to study the elasticity of biological molecules. Optical tweezers are only used to induce point forces; they are not used for imaging. They too suffer from irreproducibility when used to measure the mechanical response of cells. Why are these measurements unreliable? The problem is that the local 
mechanical response is masked by overall membrane deformations which tend to fluctuate among configurations with roughly equal energies. In both optical tweezer and AFM experiments, the sample is subjected to a point force and the resulting displacement is measured. With optical tweezers the force is exerted by a dielectric sphere attached to the sample and actuated by a laser beam; in AFM the force is exerted by the tip of a cantilever actuated by a piezoelectric drive. In relatively simple systems such as linear molecules like microtubes and DNA, the measurements may be related immediately to the elasticity of the sample. In more complicated situations, such as the deformation of a cell, it has proven difficult to extract intrinsic properties.

Nevertheless, there is a great impetus to measure the mechanical properties of cells. Over the past two decades micropipette experiments have been used to characterize the elastic properties in cells such as erythrocytes (red blood cells). This methodology has been developed by Evans and co-workers into the technique of force-spectroscopy, an important means to characterize the elastic properties of cells at the micron scale and above [4]. The associated field of biological membrane elasticity has become a very active and important area of biophysics. The model introduced in this article allows a precise description of the behavior of cell membranes under the large deformations induced by these techniques in an attempt to find new ways to characterize the health of cells, as well as a rich variety of exotic thermodynamic phenomena [5].

One goal of techniques such as liquid-cell AFM and optical tweezers is to extend the study of the mechanics of biological cells to incorporate these techniques. It permits the local elastic response (related to the intrinsic mechanical properties) to be separated from the gross cell deformation. The model is appropriate for a wide variety, albeit not all, types of cells. In particular, it is suitable for cells with a regular shape and an exterior membrane (as opposed to a cell wall). This includes cells such as erythrocytes, sperm cells and neural axons as well as organelles such as the cell nucleus and mitochondria. The model, together with the new experimental techniques, will enable a fine-grained characterization of the mechanical properties of these cells and organelles that is not currently available.

\section{Literature review}

A modern survey of the physics of membranes and the mechanisms responsible for their behavior is contained in [6,7]. A good source of general information on cell membranes is the monograph by Evans and Skalak [4]. An elementary modern review of cell mechanics is contained in the book by Boal [8]. From the mathematical modeling point of view, the first serious attempt to model the cell as an elastic surface using classical isotropic plate theory with quadratic free energy was made by Fung [9] and Fung and Tong [10]. In the physics literature, this model was reinterpreted primarily by Canham [11] and Helfrich [12], who cite Fung as the source of the model. They treat the membrane as a curved surface with energy dependent on its curvature and local stretch, but with no reference to the fluidity or solidity of the material from which the surface is made. The Fung-Canham-Helfrich model is recovered in this paper as a simple particular case, the first approximation of our model. The next step up in refining Fung's model was carried out by Ericksen $[13,14]$. His theory, based on general Cosserat surface theory, is however more complex than is necessary to model the phenomenology of interest here. Jenkins also used the concept of a fluid Cosserat surface and specialized it to obtain a specific theory which is subsumed under that considered here [15]. Numerical treatment of axisymmetric membranes are discussed by Deuling and Helfrich [16] and Jenkins [17], but these papers do not discuss the case of point loads or contact.

A general treatment of elastic membranes in which particular emphasis is placed on the distinction between solid- and fluid-like response is contained in a series of papers by Steigmann. An account of how the use of constraints lead from a general surface elasticity theory to one which incorporates the present theory as a special case is contained in [20]. This forms the basis for Steigmann [18], which contains a rationalization of the subject regarding material symmetry as it relates to the distinction between solid and fluid membranes. The models discussed thus far are particular cases of this general formulation. Further consequences of the minimum energy postulate for equilibrium in conventional (no curvature dependence) capillary systems is proved in [19] and comparisons are made with experiment. 


\section{Local constitutive response}

The mechanical response of the biological cell membrane is described by an isothermal free-energy function per unit mass, $\psi$, which depends on scalar invariants of the surface strain and curvature in a manner which reflects the fluid-like characteristics of the membrane wall. Specifically, the local response function is of the form

$\psi=\hat{\psi}(J, H, K)$,

where $J=\lambda \mu$ is the local area stretch of the membrane relative to a fixed reference surface $\Omega, H$ is the mean curvature of the membrane in its actual loaded configuration $\omega$, and $K$ is its Gaussian curvature. Here, $\lambda$ and $\mu$ are the principal stretches, the ratios of deformed to reference lengths of material curves aligned with the principal axes of surface strain. These variables are rendered explicit in Section 3 below. The fact that (1) is the most general form of the strain energy for fluid membranes is proved in $[20,21]$. The free energy functions of Fung [9], Canham [11] and Helfrich [12] are particular cases of (1).

We observe that the variable $J$ is dimensionless whereas $H$ and $K$ have dimensions of reciprocal length and squared reciprocal length, respectively; thus there is an inherent local length scale in function (1). The only such scale available is the thickness $t$ of the membrane, typically on the order of the length of the one or two polar molecules, in the case of monolayers or bilayers, respectively, which traverse the wall. This is not an explicit parameter in the theory but it is nevertheless needed to generate physically meaningful explicit forms of the response function.

To illustrate, let $E$ be a constant with dimensions of energy per unit mass. Then

$\Psi=E^{-1} \psi\left(J, \tilde{H} / t, \tilde{K} / t^{2}\right)=\Psi(J, \tilde{H}, \tilde{K})$

is a dimensionless response function in which

$\tilde{H}=t H \quad$ and $\quad \tilde{K}=t^{2} K$

are the dimensionless mean and Gaussian curvatures, respectively. Let $R$ be the (unknown) minimum radius of curvature of the membrane in its deformed state $\omega$. Then the variables in (3) are no larger than

$\tilde{H}=O(\varepsilon)$ and $\tilde{K}=O\left(\varepsilon^{2}\right)$,

respectively, where $\varepsilon=t / R$. Theories of the kind used in this work are normally reliable provided that $\varepsilon \ll 1$.
A typical guideline is that $\varepsilon$ should not exceed 0.1 . Assuming $\Psi$ to be a smooth function of the curvatures, we then obtain the estimate

$$
\begin{aligned}
\Psi(J, \tilde{H}, \tilde{K})= & \tilde{F}(J)+\tilde{A}(J) \tilde{H}+\tilde{B}(J) \tilde{H}^{2} \\
& +\tilde{C}(J) \tilde{K}+o\left(\varepsilon^{2}\right),
\end{aligned}
$$

where $o\left(\varepsilon^{2}\right) / \varepsilon^{2} \rightarrow 0$ as $\varepsilon \rightarrow 0$; the latter terms are thus negligible in comparison to those retained explicitly, provided that $\varepsilon$ is sufficiently small.

The strain energy $w$ is the free energy per unit area of the surface $\omega$ and is related to $\psi$ by

$w=\rho \psi$,

where $\rho$ is the mass per unit area (density) of the membrane in the configuration $\omega$. This is in general a positively valued function of coordinates on $\omega$. It is related to the positive mass density $\rho_{0}$ in configuration $\Omega$ by the local mass conservation equation

$\rho_{0}=\rho J$.

Unlike $\rho, \rho_{0}$ does not depend on the deformation of the membrane but may be a function of surface coordinates on $\Omega$.

Using $\rho_{0}$ we may also define the strain energy $W$ per unit area of $\Omega$. This is given by

$W=\rho_{0} \psi=J w$.

The total strain energy in a given deformation may then be represented in either of the equivalent forms

$\int_{\omega} w \mathrm{~d} a=\int_{\Omega} W \mathrm{~d} A$.

If we assume $\rho_{0}$ to be uniform, combine (8) and (6) with the dimensional version of (5), and drop the $o\left(\varepsilon^{2}\right)$ terms we derive

$$
\begin{gathered}
W=F(J)+A(J) H+B(J) H^{2}+C(J) K \quad \text { and } \\
w=f(J)+a(J) H+b(J) H^{2}+c(J) K,
\end{gathered}
$$

where $f(J)=F(J) / J, a(J)=A(J) / J$, etc. The widely used Helfrich model is the special case of $(10)_{2}$ given by

$w(J, H, K)=T-2 H_{0} H+k H^{2}+\bar{k} K$,

where $T, H_{0}, k$ and $\bar{k}$ are constants. The first of these corresponds to the function $F(J)=T J$ in $(10)_{1}$. In fact Helfrich used a slightly different form for $F(J)$ motivated by the observation that the surface tension 
of membranes typically relaxes to zero in the absence of areal stretch. This is discussed in more detail below. Originally Fung used classical isotropic plate theory to motivate a formula equivalent to (11) but later it was shown how dependence on $J, H$ and $K$ emerges from a definition of fluidity which imposes more stringent restrictions than the $S O(2)$ invariance associated with isotropy [20].

If the latter three coefficients in (11) are set to zero, we find that the total strain energy is proportional to the area of $\omega$; the proportionality constant is $T$, the surface tension. This is independent of the local dilatation $J$ and therefore (11) predicts that the membrane offers no stiffness against changes in its surface area. Although this prediction is in accord with the behavior of classical soap films and certain surfactant vesicles, it is not compatible with data on biological cells [4], which indicate that membranes are relatively stiff against dilatation. In such circumstances the derivative $F^{\prime}(J)$ is not constant. The large areal stiffness suggests that $J$ remains close to unity in typical circumstances and thus an appropriate estimate is given by Helfrich's proposal $F(J)=\frac{1}{2} E(J-1)^{2}$ where $E$ is a positive constant which may be identified with the constant used in the normalization scheme (2). This yields an effective surface tension equal to $E(J-1)$, which vanishes for $J=1$ in accordance with the understood phenomenology of cell membranes. If the dilatational modulus $E$ is sufficiently large, then deviations of $J$ from unity are energetically disfavored and it is appropriate to consider imposing the constraint $J=1$. This is discussed below. In this case we use a Lagrange multiplier to accommodate the constraint and replace $F(J)$ by $T(J-1)$, where $T$ is no longer a parameter but rather a function of coordinates on $\omega$ to be determined together with the deformation, as detailed in what follows.

Further, if $\omega$ is a closed surface then the term involving $\bar{k}$ contributes a constant to the total strain energy which is fixed by the genus of $\omega$ (roughly, the number of handles on the surface); this does not contribute to the Euler-Lagrange (equilibrium) equations and may therefore be dropped from the expression for the strain energy if the topology of the surface is fixed. It is generally necessary to retain this term for surfaces with edges.

A special case of (11) with $\bar{k}=0$ was used in [22] together with empirical AFM data on bovine sperm cells to estimate the average value of $\tilde{H}$ on $\omega$ to be on the order of $10^{-3}$. If this average value were comparable to the quantity $\varepsilon$ defined in the foregoing then the strain energy would be well approximated by (11) uniformly over $\omega$; that is, the error incurred by using a quadratic approximation would be uniformly distributed over the surface. This is not the case, however, since the AFM probe transmits what is effectively a point load to the cell, and the local curvature in the vicinity of the probe tip, which is characterized by $\varepsilon$, may be much larger than the surface average. For example, comparing the membrane thickness to the radius of curvature of the AFM probe tip yields a theoretical maximum value for $\varepsilon$ of roughly 0.3 , assuming that the radius of curvature of the membrane at the tip is equal to the tip radius. In this worst-case scenario $\varepsilon$ is still less than unity and therefore fulfills a basic necessary condition for the applicability of the theory. However, it is not to be expected that a quadratic approximation to the energy, as in (11), would furnish a good estimate locally near the probe tip. Thus, the quality of the approximation afforded by (11) deteriorates as the probe tip is approached. This fact furnishes some impetus for retaining a general dependence of the response function on $J, H$ and $K$ in the present paper. This observation notwithstanding, it may be anticipated that comparison of numerical results based on the Helfrich form with empirical shape data away from the tip may yield quantitative results sufficient to determine the various moduli appearing in the former expression.

\section{The potential energy functional for axisymmetric states}

To develop the equilibrium equations for axisymmetric states one could begin with the general equations of shell theory and then specialize, but it is more efficient to derive them directly by minimizing an appropriate potential energy, or enthalpy, functional. To this end it is necessary to write the potential energy in terms of suitable deformation functions. The desired equilibrium equations are then the Euler-Lagrange equations associated with the potential energy. This procedure is straightforward but involved and we accordingly include sufficient detail to permit our analysis to be reconstructed by the interested reader. The 
basic form of the potential energy functional used here is similar to that used in [19] for pure membranes without flexural stiffness.

We assume that the AFM probe supplies a controlled force vector $\mathbf{f}$ to the membrane at a point of $\omega$ with position $\mathbf{x}^{*}$. We further suppose that the membrane is impermeable and encloses a volume of incompressible liquid; we thus impose a global constraint on the enclosed volume. This introduces a Lagrange multiplier $p$ which is a constant to be determined in the course of solution. Physically, $p$ represents the net pressure transmitted across the membrane surface as required to enforce the constraint. Specific boundary-, continuity- and contact-conditions are discussed below and reference will also be made to the adjustments necessary to accommodate surface-area constraints of the kind often imposed in studies of cell membranes [4].

The appropriate form of the potential energy functional is $[19,23]$

$E=\int_{\Omega} W \mathrm{~d} A-p V(\omega)-\mathbf{f} \cdot \mathbf{x}^{*}$,

where the dot is used to denote the inner product of vectors and $V(\omega)$ is the volume enclosed by the membrane in its deformed configuration $\omega$. The volume constraint then takes the form

$V(\omega)=V(\Omega)$

which supplies an equation to determine $p$.

We assume that $\omega$ and $\Omega$ are surfaces of revolution with a common axis of symmetry and position functions

$\mathbf{x}(\theta, s)=x(s) \mathbf{e}_{r}(\theta)+z(s) \mathbf{k}$,

$\mathbf{X}(\theta, s)=\rho(s) \mathbf{e}_{r}(\theta)+\varsigma(s) \mathbf{k}$,

respectively, where $\theta$ is the azimuthal angle common to points on both surfaces, $s$ measures arclength along a meridian of the reference surface $\Omega, \mathbf{k}$ is a unit vector aligned with the symmetry axis, $\mathbf{e}_{r}(\theta)$ is a unit vector directed radially from the symmetry axis at azimuth $\theta$, and $(x, z),(\rho, \varsigma)$ with $x \geqslant 0$ and $\rho \geqslant 0$ are the radial and axial coordinates of a point of the membrane on $\omega$ and $\Omega$, respectively. These parametrizations may be used to plot the points of the meridian in the $\mathbf{e}_{r}, \mathbf{k}$-plane and therefore furnish a convenient means to visualize the solution graphically.
We define $\theta^{1}=\theta, \theta^{2}=s$ and use these as surface coordinates throughout the following development. They may be used to define vectors

$$
\begin{aligned}
& \mathbf{a}_{1}=\mathbf{x}_{, 1}=x(s) \mathbf{e}_{\theta}(\theta) \quad \text { and } \\
& \quad \mathbf{a}_{2}=\mathbf{x}_{, 2}=x^{\prime}(s) \mathbf{e}_{r}(\theta)+z^{\prime}(s) \mathbf{k},
\end{aligned}
$$

where $\mathbf{e}_{\theta}=\mathbf{k} \times \mathbf{e}_{r}$ and the commas followed by subscripts indicate differentiation with respect to the indicated coordinates. These vectors are tangential to the circles of latitude and meridians on $\omega$, respectively. Their counterparts on $\Omega$ are given by

$$
\begin{aligned}
& \mathbf{A}_{1}=\mathbf{X}_{, 1}=\rho(s) \mathbf{e}_{\theta}(\theta) \quad \text { and } \\
& \quad \mathbf{A}_{2}=\mathbf{X}_{, 2}=\rho^{\prime}(s) \mathbf{e}_{r}(\theta)+\varsigma^{\prime}(s) \mathbf{k} .
\end{aligned}
$$

The definition of $s$ as arclength along a meridian of $\Omega$ implies that $\left(\rho^{\prime}\right)^{2}+\left(\varsigma^{\prime}\right)^{2}=1$ and therefore $\left|\mathbf{A}_{2}\right|=1$.

The exterior unit normal to $\omega$ is

$$
\begin{aligned}
\mathbf{n} & =\mathbf{a}_{1} \times \mathbf{a}_{2} /\left|\mathbf{a}_{1} \times \mathbf{a}_{2}\right| \\
& =\left(z^{\prime} \mathbf{e}_{r}-x^{\prime} \mathbf{k}\right) / \sqrt{\left(x^{\prime}\right)^{2}+\left(z^{\prime}\right)^{2}}
\end{aligned}
$$

and is used to obtain a useful expression for the volume enclosed by $\omega$. First we write

$V(\omega)=\frac{1}{3} \int_{V(\omega)} \operatorname{div} \mathbf{x} \mathrm{d} v$,

where $\mathbf{x}$ is the position of points inside the surface, the extension of $(14)_{1}$ to the interior, and $\operatorname{div} \mathbf{x}(=3)$ is its divergence. We then use the divergence theorem to obtain

$V(\omega)=\frac{1}{3} \int_{\omega} \mathbf{x} \cdot \mathbf{n} \mathrm{d} a=\frac{1}{3} \int_{\Omega} J \mathbf{x} \cdot \mathbf{n} \mathrm{d} A$,

where $\mathbf{x}$ and $\mathbf{n}$ are given by (14) and (17), respectively. The factor $J$ is related to the deformation variables by writing $J=\lambda \mu$ where

$$
\begin{gathered}
\lambda=\left|\mathbf{a}_{2}\right| /\left|\mathbf{A}_{2}\right|=\sqrt{\left(x^{\prime}\right)^{2}+\left(z^{\prime}\right)^{2}} \quad \text { and } \\
\mu=\left|\mathbf{a}_{1}\right| /\left|\mathbf{A}_{1}\right|=x / \rho
\end{gathered}
$$

are the stretches of the meridians and circles of latitude, respectively; therefore

$J=(x / \rho) \sqrt{\left(x^{\prime}\right)^{2}+\left(z^{\prime}\right)^{2}}$

and

$$
\begin{aligned}
J \mathbf{n} \cdot \mathbf{x} & =(x / \rho)\left(x z^{\prime}-z x^{\prime}\right) \\
& =\rho^{-1}\left[x^{2} z^{\prime}-z\left(\frac{1}{2} x^{2}\right)^{\prime}\right] .
\end{aligned}
$$


The expression for $J$ is also needed in the strain-energy function. To obtain the curvature terms in this function we use [24]

$$
\begin{aligned}
2 H & =a^{\alpha \beta} b_{\alpha \beta} \quad \text { and } \\
K & =\operatorname{det}\left(b_{\alpha \beta}\right) / \operatorname{det}\left(a_{\alpha \beta}\right),
\end{aligned}
$$

where, in the formula for $H$, repeated indices are summed from 1 to 2 and

$$
\begin{gathered}
a_{\alpha \beta}=\mathbf{a}_{\alpha} \cdot \mathbf{a}_{\beta}, \quad b_{\alpha \beta}=\mathbf{n} \cdot \mathbf{a}_{\alpha, \beta} \quad \text { and } \\
\left(a^{\alpha \beta}\right)=\left(a_{\alpha \beta}\right)^{-1} .
\end{gathered}
$$

A lengthy but straightforward calculation yields

$$
\begin{aligned}
2 H= & \left(z^{\prime} x^{\prime \prime}-x^{\prime} z^{\prime \prime}\right) /\left[\left(x^{\prime}\right)^{2}+\left(z^{\prime}\right)^{2}\right]^{3 / 2} \\
& -x^{-1} z^{\prime} /\left[\left(x^{\prime}\right)^{2}+\left(z^{\prime}\right)^{2}\right]^{1 / 2}
\end{aligned}
$$

and

$$
K=x^{-1} z^{\prime}\left(x^{\prime} z^{\prime \prime}-z^{\prime} x^{\prime \prime}\right) /\left[\left(x^{\prime}\right)^{2}+\left(z^{\prime}\right)^{2}\right]^{2} .
$$

Therefore the strain-energy function may be written in the form

$$
W(J, H, K)=\bar{W}\left(x, x^{\prime}, x^{\prime \prime} ; z, z^{\prime}, z^{\prime \prime} ; s\right) \text {, }
$$

where the explicit $s$-dependence enters through the function $\rho(s)$ pertaining to the reference surface.

It is advantageous to write the entire potential energy $E$ as an integral over $\Omega$ and to this end we let $s$ range over $(a, b)$ where $b$ is the value of arclength at the pole where the point load is applied. If the load is directed vertically along the symmetry axis then $\mathbf{f} \cdot \mathbf{x}^{*}=F z(b)$ where $F$ is the scalar force, negative if the force is directed downward. We then use $z(b)=z(a)+\int_{a}^{b} z^{\prime}(s) \mathrm{d} s$ together with $\mathrm{d} A=2 \pi \rho(s) \mathrm{d} s$ to obtain

$E / 2 \pi=\int_{a}^{b} G\left(x, x^{\prime}, x^{\prime \prime} ; z, z^{\prime}, z^{\prime \prime} ; s\right) \mathrm{d} s$,

where

$$
\begin{aligned}
G= & \rho(s) \bar{W}\left(x, x^{\prime}, x^{\prime \prime} ; z, z^{\prime}, z^{\prime \prime} ; s\right) \\
& -\frac{1}{3} p\left[x^{2} z^{\prime}-z\left(\frac{1}{2} x^{2}\right)^{\prime}\right]-\bar{F} z^{\prime}
\end{aligned}
$$

and $\bar{F}=F / 2 \pi$. For the problems considered here we prescribe $z(a)$ and neglect the term $F z(a)$ which contributes only a fixed constant to the energy and therefore does not contribute to the equilibrium equations.
The functional (29) is in standard form and the Euler-Lagrange equations may be deduced by appealing to results given in [23]. They are:

$$
\begin{gathered}
\left(G_{x^{\prime \prime}}\right)^{\prime \prime}-\left(G_{x^{\prime}}\right)^{\prime}+G_{x}=0 \quad \text { and } \\
\left(G_{z^{\prime \prime}}\right)^{\prime \prime}-\left(G_{z^{\prime}}\right)^{\prime}+G_{z}=0,
\end{gathered}
$$

where subscripts refer to partial derivatives. A novel feature of the present formulation is the incorporation of the point load directly into the differential equations through the definition of $G$. After substituting (29) we arrive at a coupled pair of non-linear fourth-order ordinary differential equations for $x(s)$ and $z(s)$. To obtain these equations in explicit form it is necessary to use the chain rule together with the particular strain-energy function $W$ under consideration. For example,

$G_{x}=\rho \bar{W}_{x}-\frac{1}{3} p\left(2 x z^{\prime}-z x^{\prime}\right)$,

where

$\bar{W}_{x}=W_{J} J_{x}+W_{H} H_{x}+W_{K} K_{x}$

and $J_{x}, H_{x}, K_{x}$ are obtained from (30)-(32) once and for all.

The boundary conditions are also obtained by appealing to standard variational arguments. Thus, let $u(s)$ and $v(s)$ be the variations of the functions $x(s)$ and $z(s)$. These are the Gateaux derivatives of $x$ and $z$ with respect to a parameter which labels geometrically feasible configurations of the membrane. They may be viewed as the velocities of these functions with respect to the parameter and the boundary conditions may then be deduced from the virtual-work conditions [23]

$$
\begin{aligned}
& u(a)\left[G_{x^{\prime}}-\left(G_{x^{\prime \prime}}\right)^{\prime}\right]_{a}=0 ; \quad u(b)\left[G_{x^{\prime}}-\left(G_{x^{\prime \prime}}\right)^{\prime}\right]_{b}=0, \\
& v(a)\left[G_{z^{\prime}}-\left(G_{z^{\prime \prime}}\right)^{\prime}\right]_{a}=0 ; \\
& v(b)\left[G_{z^{\prime}}-\left(G_{z^{\prime \prime}}\right)^{\prime}\right]_{b}=0
\end{aligned}
$$

and

$u^{\prime}(a)\left[G_{x^{\prime \prime}}\right]_{a}=0, \quad u^{\prime}(b)\left[G_{x^{\prime \prime}}\right]_{b}=0$,

$v^{\prime}(a)\left[G_{z^{\prime \prime}}\right]_{a}=0, \quad v^{\prime}(b)\left[G_{z^{\prime \prime}}\right]_{b}=0$.

The bracketed expressions multiplying $u$ and $v$ are forces in the $x$ and $z$ directions, respectively, the terms multiplying $u^{\prime}$ and $v^{\prime}$ are couples.

This framework encompasses a number of boundary-value problems. We consider two examples here and then proceed to discuss adjustments 
to the theory needed to accommodate unilateral contact conditions and local area constraints.

First, suppose the membrane has the shape of a lens in its reference configuration and let it be pinned to a circle of radius $A$ on the plane $z=\varsigma=C$.

Take the point $s=a$ on the meridian to correspond to a point on the plane. Then $x(a)=A, z(a)=C$ and so $u(a)=0$ and $v(a)=0$. Eqs. (33) $)_{1}$ and (34) $)_{1}$ are automatically satisfied. At the pole $s=b$ the tangent to the deformed meridian is horizontal and therefore $\mathrm{d} z / \mathrm{d} x=0$ there. Writing this as the ratio $z^{\prime}(s) / x^{\prime}(s)$ we obtain $z^{\prime}(b)=0$ and therefore $v^{\prime}(b)=0$. The radius at the pole also vanishes, i.e. $x(b)=0$ and thus $u(b)=0$. The remaining four boundary conditions are

$$
\begin{aligned}
& G_{z^{\prime}}-\left(G_{z^{\prime \prime}}\right)^{\prime}=0 \quad \text { at } s=b, \\
& G_{x^{\prime \prime}}=0, \quad G_{z^{\prime \prime}}=0 \quad \text { at } s=a \quad \text { and } \\
& \quad G_{x^{\prime \prime}}=0 \quad \text { at } s=b .
\end{aligned}
$$

The first of these requires that the net vertical force acting at the pole vanish; the remaining equations require that appropriate bending moments vanish at the points indicated. The formulation is completed by specifying a parametrization of the reference surface $\Omega$. This problem is relevant to bovine sperm cells which are relatively flattened surfaces in the absence of AFM loads. We would then identify such a configuration with the reference surface $\Omega$.

As a general observation we note that if the reference configuration chosen is intended to be an actual configuration in the absence of the AFM load, then the equilibrium equations with $F=0$ must be satisfied when $x(s)$ and $z(s)$ are replaced by $\rho(s)$ and $\varsigma(s)$, respectively, the latter cannot then be specified arbitrarily.

If the reference surface is a sphere under opposing axial point loads, then we may invoke the symmetry of the problem with respect to the equatorial plane. Here we impose $z(a)=0$ where $s=a$ corresponds to the point on the meridian at the equator. Thus $v(a)=0$ and of course $x(b)=0$ and $u(b)=0$. In the deformed configuration the tangent to the meridian will be vertical at the equator and horizontal at the pole. Thus $x^{\prime}(a)=z^{\prime}(b)=0$ and so $u^{\prime}(a)=v^{\prime}(b)=0$. The remaining four boundary conditions are

$$
\begin{gathered}
G_{x^{\prime}}-\left(G_{x^{\prime \prime}}\right)^{\prime}=0 \quad \text { at } s=a \quad \text { and } \\
G_{z^{\prime}}-\left(G_{z^{\prime \prime}}\right)^{\prime}=0 \quad \text { at } s=b
\end{gathered}
$$

together with

$$
\begin{gathered}
G_{x^{\prime \prime}}=0 \quad \text { at } s=b \quad \text { and } \\
G_{z^{\prime \prime}}=0 \quad \text { at } s=a .
\end{gathered}
$$

In general, for closed membranes subject to a volume constraint the pressure $p$ in the foregoing equations plays the role of a Lagrange multiplier which must be determined in the course of solution. To this end it is necessary to first assign a value of $p$ together with the specified data and then compute a solution curve $(x(s ; p), z(s ; p))$ representing the meridian of the equilibrated membrane, depending parametrically on $p$. This may be accomplished by using any number of well-established techniques for solving systems of ordinary differential equations. The computed solution is then used to define a function

$$
\begin{aligned}
I(p)= & \int_{a}^{b}\left[x^{2} z^{\prime}-z\left(\frac{1}{2} x^{2}\right)^{\prime}\right] \mathrm{d} s \\
& -\int_{a}^{b}\left[\rho^{2} \varsigma^{\prime}-\varsigma\left(\frac{1}{2} \rho^{2}\right)^{\prime}\right] \mathrm{d} s
\end{aligned}
$$

which vanishes whenever constraint (13) is satisfied. Of course the values of $p$ used cannot be expected to satisfy $I(p)=0$ and so it is necessary to select a sequence of values $\left\{p_{i}\right\}_{i=1, \ldots, n}$ and to repeat the solution process for every member of the sequence. This generates a sequence of values $I\left(p_{i}\right)$ which may then be plotted and by using an interval-splitting method it is possible to obtain an estimate of the correct value of $p$ to arbitrary accuracy. The associated functions $(x(s), z(s))$ then furnish a parametric representation of the meridian curve of the actual membrane. The same procedure may be used to generate multiple solutions in the event of non-uniqueness, which cannot be ruled out a priori. Existence of solutions may be demonstrated by adapting Peano's well-known classical existence theorem for systems of ordinary differential equations, which in turn forms the basis of numerical methods such as the so-called shooting technique. It is of course necessary to use numerical quadrature to evaluate $I(p)$.

We may generalize the problem in at least two important directions which bear directly on the simulation of cell-membrane response. In the first generalization, we impose a local constraint on the area of the membrane in accordance with observed cell-membrane phenomenology as reported in the 
monograph by Evans and Skalak [4], for example. Experiments indicate that it is energetically favorable for red-blood cell membranes to deform in such a way to maintain local surface area whenever such a mode of deformation is kinematically compatible with boundary data. When this is not feasible, closed impermeable membranes continue to respect the enclosed volume constraint, suggesting that there is a substantially greater energetic penalty associated with variations in volume than with variations in area [4]. In the latter circumstances the foregoing formulation furnishes the appropriate model.

To accommodate the area constraint, we impose the local subsidiary condition $J=1$, which yields the non-holonomic (differential) condition

$\left(x^{\prime}\right)^{2}+\left(z^{\prime}\right)^{2}=(\rho / x)^{2}$.

This must be satisfied in addition to Euler-Lagrange equations (30). Energy functional (28) continues to apply but in expression (29) for the strain-energy function, the constant $T$, which represents a material parameter in the unconstrained case, is replaced by a function $T(s)(J-1)$ of the reference arclength variable [23]. The function $T(s)$, which is unrelated to the local material properties, is added to the list of unknowns $x(s), z(s)$. With this modification, the EulerLagrange equations and the various associated boundary conditions remain valid, and together with (33) and (34) furnish a complete system for the determination of $x(s), z(s), T(s)$. The solution procedure outlined in the foregoing remains valid also, but now the functions $x(s ; p), z(s ; p), T(s ; p)$ thereby obtained depend parametrically on $p$, which is determined as before by requiring that $I(p)$ vanish. Of course, when imposing simultaneous constraints on local area and global volume it may happen that the boundary data or other conditions of the problem imply that there is no nontrivial solution. The question turns on the existence of a non-trivial intersection of the classes of functions meeting (40) and $I=0$ simultaneously. If there is no non-trivial intersection (the trivial one being that corresponding to the reference surface) then the work of Evans and Skalak [4] indicates that the area constraint should be relaxed.

Lastly, in the second generalization, the membrane is modelled as a closed surface in contact with a horizontal plane rigid substrate. We no longer have symmetry with respect to an equatorial plane and therefore replace the boundary conditions by appropriate continuity conditions. Specifically, we take the point $s=a$ to correspond to the point on the contact plane which also lies on the vertical symmetry axis. The point $s=b$ is the arclength station of the point where the AFM tip probe is applied, as before. The tip load causes the membrane to come into contact with the substrate over an interval $a<s<c$ of the meridian, where $c$ is an unknown parameter which must be determined in the course of solution. In the (unknown) region of contact, we impose the unilateral constraint $z(s) \geqslant 0$ on kinematically possible configurations of the membrane. If the contact is frictionless, then the problem remains conservative and admits the same potentialenergy functional that we have used thus far. Using techniques from the calculus of variations in the presence of unilateral constraints, it may then be shown that in the region of contact, a one-signed Lagrange multiplier function $q(s)$ is operative, and plays the role of a (variable) lateral pressure which acts in addition to the uniform pressure $p$ associated with the volume constraint. The foregoing Euler-Lagrange equations (30), together with (33) and (34) if appropriate, remain valid everywhere on the meridian from $s=a$ to $b$, but in the region $a<s<c$, the pressure $p$ in these equations is replaced by $p+q(s)$. This would appear to introduce a surplus of unknown functions into the formulation, but in fact we impose the condition $z(s)=0 ; a<s<c$, in the Euler-Lagrange equations so that the nominal unknown $z(s)$ is effectively replaced by the unknown $q(s)$ in the contact region; thus we again have two equations (or three in the presence of the area constraint), and hence a system sufficient to determine the unknown functions.

To determine the parameter $c$ which defines the part of the membrane in contact with the plane, we impose the requirement

$q(c)=0$,

so that the additional contact pressure vanishes at the edge of the contact domain (and of course elsewhere on the membrane), together with continuity conditions on the meridional position and slope fields; thus

$$
\begin{aligned}
& x_{\mathrm{n}}(c)=x_{\mathrm{c}}(c), \quad z_{\mathrm{n}}(c)=z_{\mathrm{c}}(c)=0 ; \\
& x_{\mathrm{n}}^{\prime}(c)=x_{\mathrm{c}}^{\prime}(c), \quad z_{\mathrm{n}}^{\prime}(c)=z_{\mathrm{c}}^{\prime}(c)=0,
\end{aligned}
$$

where $x_{\mathrm{n}}(s), z_{\mathrm{n}}(s)$ are the coordinates of points on the meridian in regions where there is no contact and $x_{\mathrm{c}}(s)$, 
$z_{\mathrm{c}}(s)=0$ are the coordinates in the contact region. The complete meridian is then given by the composite functions

$$
\begin{aligned}
x(s)=\begin{array}{ll}
x_{\mathrm{c}}(s), & a \leqslant s<c, \\
x_{\mathrm{n}}(s), & c \leqslant s<b,
\end{array} \\
z(s)=\begin{array}{ll}
0, & a \leqslant s<c, \\
z_{\mathrm{n}}(s), & c \leqslant s \leqslant b .
\end{array}
\end{aligned}
$$

The foregoing solution technique for the determination of the Lagrange multiplier $p$ remains valid with or without the local area constraint. However, for every choice of $p$ in the sequence of solutions, it is necessary to adjust the parameter $c$ until $q(c)=0$ is satisfied. All functions and parameters, including $c$, depend parametrically on $p$, and the function $I(p)$, whose zeros identify solutions to the equilibrium problem, may then be constructed and used as before.

The methodology outlined in this paper, although straightforward, is nevertheless computationally intensive and therefore specific examples will be discussed elsewhere.

\section{Acknowledgements}

This work was performed under the auspices of the US Department of Energy by the University of California, Lawrence Livermore National Laboratory, under Contract No. W-7405-Eng-48. The authors wish to acknowledge R. Balhorn and M. Allen for many fruitful discussions. E. Baesu wishes to express her gratitude for the hospitality of members of the Materials Research Institute at LLNL during her visit in the summer of 2001.

\section{References}

[1] V.J. Morris, A.P. Gunning, A.R. Kirby, Atomic Force Microscopy for Biologists, Imperial College Press, London, 1999.

[2] A. Ashkin, Optical trapping and manipulation of neutral particles using lasers, Proc. Nat. Acad. Sci. 94 (1997) 4853.

[3] E. A-Hassan, W.F. Heinz, M.D. Antonik, N.P. D'Costa, S. Nageswaran, C.A. Schoenenberger, J.H. Hoh, Relative microelastic mapping of living cells by atomic force microscopy, Biophys. J. 74 (1998) 1564.

[4] E.A. Evans, R. Skalak, Mechanics and Thermodynamics of Biomembranes, CRC Press, Boca Raton, FL, 1980.
[5] D. Nelson, T. Piran, S. Weinberg (Eds.), Statistical Mechanics of Membranes and Surfaces, World Scientific, Singapore, 1989.

[6] H. Kellay, B.P. Binks, Y. Hendrikx, L.T. Lee, J. Meunier, Properties of surfactant monolayers in relation to microemulsion phase behaviour, Adv. Colloid Interface Sci. 49 (1994) 85.

[7] W.M. Gelbart, A. Ben-Shaul, D. Roux (Eds.), Micelles, Membranes, Microemulsions, and Monolayers, Springer Series on Partially Ordered Systems, Springer, New York, 1994.

[8] D.H. Boal, Mechanics of Cells, Cambridge University Press, Cambridge, 2002.

[9] Y.C. Fung, Theoretical considerations of the elasticity of red blood cells and small blood vessels, Proc. Fed. Am. Soc. Exp. Biol. 25 (1966) 1761.

[10] Y.C. Fung, P. Tong, Theory of sphering of red blood cells, Biophys. J. 8 (1968) 175.

[11] P.B. Canham, The minimum energy as possible explanation of the biconcaveshape of the human red blood cell, J. Theoret. Biol. 26 (1970) 61.

[12] W. Helfrich, Elastic properties of lipid bilayers: theory and possible experiments, Z. Naturforsch. 28c (1973) 693.

[13] J.L. Ericksen, Liquid crystals and Cosserat surfaces, Q. J. Mech. Appl. Math. 27 (1974) 213.

[14] J.L. Ericksen, Theory of Cosserat surfaces and its applications to shells, interfaces and cell membranes, in: P.G. Glockner, M. Epstein, D.J. Malcolm (Eds.), Proceedings of the International Symposium on Recent Developments in the Theory and Application of Generalized and Oriented Media, Vol. 27, 1979.

[15] J.T. Jenkins, The equations of mechanical equilibrium of a model membrane, SIAM J. Appl. Math. 32 (1977) 755.

[16] H.J. Deuling, W. Helfrich, The curvature elasticity of fluid membranes: a catalogue of vesicle shapes, J. Phys. 37 (1976) 1335.

[17] J.T. Jenkins, Static equilibrium configurations of a model red blood cell, J. Math. Biol. 4 (1977) 149.

[18] D.J. Steigmann, On the relationship between the Cosserat and Kirchhoff- Love theories of elastic shells, Math. Mech. Solids 4 (1999) 275.

[19] D.J. Steigmann, D. Li, A theory of elasticity for fluid films, Proc. R. Soc. London A 449 (1995) 223.

[20] D.J. Steigmann, Fluid films with curvature elasticity, Arch. Rational Mech. Anal. 150 (1999) 127.

[21] D.J. Steigmann, Irreducible function bases for simple fluid and liquid crystal films, J. Math. Phys. Solids (ZAMP), to appear.

[22] M. McElfresh, E. Baesu, R. Ballhorn, J. Belak, M.J. Alien, R.E. Rudd, Combining constitutive materials modeling with Atomic Force Microscopy to understand the mechanical properties of living cells, Proc. Nat. Acad. Sci. 99 (2002) 6493.

[23] I.M. Gelfand, S.V. Fomin, Calculus of Variations, Prentice-Hall, New York, 1963.

[24] P.M. Naghdi, The Theory of Plates and Shells, Handbuch der Physik, Vol. VIa/2, Springer, New York, 1972. 